\title{
Effective Interactions and Volume Energies in Charge-Stabilized Colloidal Suspensions
}

\author{
A R Dentonf \\ Department of Physics, Acadia University, Wolfville, NS, Canada B0P 1X0
}

\begin{abstract}
Charge-stabilized colloidal suspensions can be conveniently described by formally reducing the macroion-microion mixture to an equivalent one-component system of pseudo-particles. Within this scheme, the utility of a linear response approximation for deriving effective interparticle interactions has been demonstrated [M. J. Grimson and M. Silbert, Mol. Phys. 74, 397 (1991)]. Here the response approach is extended to suspensions of finite-sized macroions and used to derive explicit expressions for (1) an effective electrostatic pair interaction between pseudomacroions and (2) an associated volume energy that contributes to the total free energy. The derivation recovers precisely the form of the DLVO screened-Coulomb effective pair interaction for spherical macroions and makes manifest the important influence of the volume energy on thermodynamic properties of deionized suspensions. Excluded volume corrections are implicitly incorporated through a natural modification of the inverse screening length. By including nonlinear response of counterions to macroions, the theory may be generalized to systematically investigate effective manybody interactions.
\end{abstract}

PACS numbers: 82.70.Dd, 83.70.Hq, 05.20.Jj, 05.70.-a

Submitted to: J. Phys.: Condens. Matter

† e-mail address: alan.denton@acadiau.ca 


\section{Introduction}

Charge-stabilized colloidal suspensions, composed of charged macroions $(1-1000 \mathrm{~nm}$ in diameter) and microions (counterions and salt ions) suspended by Brownian motion in a molecular fluid, occur in a variety of natural and engineered forms [1]. Common examples include clay minerals, paints, inks, and detergents (micellar solutions), in which repulsive electrostatic interactions promote stability against coagulation induced by van der Waals attractive forces. Somewhat more exotic are suspensions of synthetic latex or silica spheres whose near monodispersity facilitates self-assembly into crystalline lattices [2]. Aside from serving as well-characterized models for fundamental study, synthetic colloids exhibit unique optical properties that underly several emerging technological applications, such as nanosecond optical switches [3] and photonic band gap materials [1].

Accurate prediction of the physical properties of colloidal matter relies on a fundamental understanding of interparticle interactions. The first quantitative account of electrostatic interactions was achieved by Derjaguin, Landau, Verwey, and Overbeek (DLVO) [5]. Based on the Poisson-Boltzmann equation for the electrostatic potential [1], the DLVO theory portrays the bare Coulomb interactions between macroions as effectively screened by a surrounding atmosphere of microions. The resulting screenedCoulomb pair potential has been a valuable cornerstone of colloid science for half a century. Nevertheless, experimental evidence for apparent long-range attractions between macroions [6] has contributed to renewed interest in colloidal interparticle interactions.

An explicit description of the multi-component mixture of macroions, counterions, salt ions, and solvent molecules clearly poses a formidable challenge. Consequently, interactions in such complex systems are usually treated at the level of effective interactions. Tracing out from the partition function statistical degrees of freedom of all but a single component reduces the problem to that of an equivalent one-component system of "pseudo-particles" governed by an effective thermodynamic-state-dependent interaction [7]. A range of theoretical and numerical methods have been developed to investigate effective interactions in charge-stabilized colloids. Strategies deployed to date include Poisson-Boltzmann cell models [8], Monte Carlo simulation [9, 10], $a b$ initio simulation [11, and density-functional theory [12, 13, 14, 15]. Recently, Silbert and coworkers [16, 17] proposed an approach motivated by analogies between charged colloids and metals. With the correspondences (counterion $\leftrightarrow$ electron) and (macroion $\leftrightarrow$ metallic ion), the procedure is closely akin to the pseudopotential theory of metals [18], which can successfully account for thermodynamic properties of simple metals [19]. Performing a classical trace over microion degrees of freedom and describing the electrostatic response of the microions to the macroions within second-order perturbation theory, results in an effective interaction between pseudomacroions and an associated volume energy that contributes to the total free energy. The importance of including the volume energy in calculating thermodynamic properties 
of charge-stabilized colloidal suspensions has been emphasized recently by a number of authors [12, 13, 14, 16, 20].

The main purpose of this paper is to develop further the response approach to charge-stabilized colloids, extending it, in particular, to suspensions of finite-sized macroions. The proposed extensions enforce exclusion of microions from the macroion hard cores and explicitly take into account the volume excluded by the macroions to the microions. A second goal of the paper is to establish a framework for generalizing the theory to include nonlinear response to allow investigation of effective many-body interactions. In the next section, following a brief review of the response approach, the extensions are outlined. Section 3 presents the main results - obtained within a linear response approximation - for an effective pair potential acting between pseudomacroions and an associated volume energy, both of which consistently incorporate excluded volume effects. Finally in Sec. 4, we discuss implications of the results for thermodynamic properties of charge-stabilized colloids and prospects for generalizing the theory beyond linear response.

\section{Theory}

\subsection{The Model}

The theory described below is based on the "primitive" model, wherein for simplicity the solvent is treated as a uniform dielectric continuum. To simplify notation, it is furthermore assumed that counterions are the only microions present (deionized suspension). The general case of finite salt concentration will be addressed elsewhere [21]. The model system then consists of $N_{\mathrm{m}}$ charged hard-sphere macroions of diameter $\sigma$ and charge $-Z e$ ( $e$ being the elementary charge) and $N_{\mathrm{c}}$ point counterions of charge ze suspended in a uniform fluid medium characterized entirely by a dielectric constant $\epsilon$. Each macroion is assumed to carry a fixed charge, uniformly distributed over its surface. Charge fluctuations are thus implicitly ignored in the model. Described statistically by a canonical ensemble, the system occupies a fixed total volume $V$ at temperature $T$. For a given number of macroions, global charge neutrality constrains the number of counterions by the condition $z N_{\mathrm{c}}=Z N_{\mathrm{m}}$.

Denoting macroion and counterion positions by $\mathbf{R}_{i}$ and $\mathbf{r}_{i}$, respectively, the full Hamiltonian of the system can be expressed in the form

$$
H=H_{\mathrm{m}}+H_{\mathrm{c}}+H_{\mathrm{mc}},
$$

with the individual terms to be specified below. The first term on the right side of Eq. (1) is the bare macroion Hamiltonian, given by

$$
H_{\mathrm{m}}=K_{\mathrm{m}}+\frac{1}{2} \sum_{\substack{i, j=1 \\ i \neq j}}^{N_{\mathrm{m}}}\left[v_{\mathrm{HS}}\left(\left|\mathbf{R}_{i}-\mathbf{R}_{j}\right|\right)+v_{\mathrm{mm}}\left(\left|\mathbf{R}_{i}-\mathbf{R}_{j}\right|\right)\right],
$$

where $K_{\mathrm{m}}$ is the kinetic energy of the macroions, $v_{\mathrm{HS}}\left(\left|\mathbf{R}_{i}-\mathbf{R}_{j}\right|\right)$ is a hard-sphere pair interaction between the macroion cores, and $v_{\mathrm{mm}}(r)=Z^{2} e^{2} / \epsilon r$ is the bare Coulomb 
interaction between a pair of macroions whose centres are separated by a distance $r>\sigma$. The second term in Eq. (1) is a counterion Hamiltonian, taking the form

$$
H_{\mathrm{c}}=K_{\mathrm{c}}+\frac{1}{2} \sum_{\substack{i, j=1 \\ i \neq j}}^{N_{\mathrm{c}}} v_{\mathrm{cc}}\left(\left|\mathbf{r}_{i}-\mathbf{r}_{j}\right|\right)+\sum_{i=1}^{N_{\mathrm{c}}} \sum_{j=1}^{N_{\mathrm{m}}} v_{\mathrm{HS}}\left(\left|\mathbf{r}_{i}-\mathbf{R}_{j}\right|\right),
$$

where $K_{\mathrm{c}}$ is the counterion kinetic energy, $v_{\text {cc }}(r)=z^{2} e^{2} / \epsilon r$ is the Coulomb interaction between a pair of counterions, and $v_{\mathrm{HS}}\left(\left|\mathbf{r}_{i}-\mathbf{R}_{j}\right|\right)$ is the hard-sphere interaction between a point counterion and a macroion core. Finally, the third term in Eq. (四) is the electrostatic interaction energy between the macroions and counterions:

$$
H_{\mathrm{mc}}=\sum_{i=1}^{N_{\mathrm{c}}} \sum_{j=1}^{N_{\mathrm{m}}} v_{\mathrm{mc}}\left(\left|\mathbf{r}_{i}-\mathbf{R}_{j}\right|\right)
$$

where $v_{\text {mc }}(r)$ denotes the macroion-counterion electrostatic pair interaction. Outside the macroion core radius, $v_{\mathrm{mc}}(r)$ has the Coulomb form. Inside the core, however, $v_{\mathrm{mc}}(r)$ is not uniquely defined. Thus, following van Roij and Hansen [12], we choose $v_{\mathrm{mc}}(r)$ to be a constant for $r<\sigma$ and take

$$
v_{\mathrm{mc}}(r)= \begin{cases}\frac{-Z z e^{2}}{\epsilon r}, & r>\sigma / 2 \\ \frac{-Z z e^{2}}{\epsilon \sigma / 2} \alpha, & r<\sigma / 2\end{cases}
$$

where the parameter $\alpha$ will be specified (Sec. 3.1) to ensure that the counterion density vanishes within the core, as physically it must.

\subsection{Reduction to an Equivalent One-Component System}

Having defined the Hamiltonian, we now turn to a statistical mechanical description, with the ultimate aim of calculating the free energy of the system. The partition function is given by

$$
\mathcal{Z}_{N}=\left\langle\left\langle\exp \left(-H / k_{\mathrm{B}} T\right)\right\rangle_{\mathrm{c}}\right\rangle_{\mathrm{m}}
$$

the angular brackets symbolizing classical traces over macroion or counterion degrees of freedom. Following standard treatments developed first in the context of simple metals [19, 22], we proceed by reducing the two-component mixture of macroions and counterions to an equivalent one-component system. The reduction is achieved by performing a restricted trace over counterion coordinates, while keeping the macroion coordinates fixed. Thus, without approximation in this purely classical system,

$$
\mathcal{Z}_{N}=\left\langle\exp \left(-H_{\mathrm{eff}} / k_{\mathrm{B}} T\right)\right\rangle_{\mathrm{m}}
$$

where $H_{\text {eff }} \equiv H_{\mathrm{m}}+F_{\mathrm{c}}$ is the effective Hamiltonian of a one-component system of pseudomacroions, and where

$$
F_{\mathrm{c}} \equiv-k_{\mathrm{B}} T \ln \left\langle\exp \left[-\left(H_{\mathrm{c}}+H_{\mathrm{mc}}\right) / k_{\mathrm{B}} T\right]\right\rangle_{\mathrm{c}}
$$

may be physically interpreted as the free energy of a nonuniform gas of counterions in the presence of macroions fixed at positions $\mathbf{R}_{i}$. In general, the counterion free energy 
is a complicated many-body function of the macroion positions. Progress can be made, however, by formally adding to and substracting from $H$ a term, $E_{\mathrm{b}}$, representing the energy of a uniform compensating negative background. Then $F_{\mathrm{c}}$ may be expressed in the form

$$
F_{\mathrm{c}}=-k_{\mathrm{B}} T \ln \left\langle\exp \left[-\left(H_{\mathrm{c}}^{\prime}+H_{\mathrm{mc}}^{\prime}\right) / k_{\mathrm{B}} T\right]\right\rangle_{\mathrm{c}},
$$

where $H_{\mathrm{c}}^{\prime} \equiv H_{\mathrm{c}}+E_{\mathrm{b}}$ and $H_{\mathrm{mc}}^{\prime} \equiv H_{\mathrm{mc}}-E_{\mathrm{b}}$. The advantage of this manipulation is that $H_{\mathrm{c}}^{\prime}$ is simply the Hamiltonian of a classical one-component plasma (OCP) of counterions in the presence of neutral hard-sphere macroions.

Now the counterions, being excluded by the hard cores of the macroions, occupy the free volume $V_{\mathrm{f}} \equiv V-N_{\mathrm{m}}(\pi / 6) \sigma^{3}$, i.e., the volume not occupied by the macroion cores. The average effective density of counterions is therefore given by $n_{\mathrm{c}}=N_{\mathrm{c}} / V_{\mathrm{f}}=$ $n_{\mathrm{c}}^{(o)} /(1-\eta)$, where $n_{\mathrm{c}}^{(o)} \equiv N_{\mathrm{c}} / V$ is the nominal counterion density and $\eta \equiv\left(V-V_{\mathrm{f}}\right) / V$ is the macroion volume fraction. An important question now arises. What volume should the background occupy? In order that $H_{\mathrm{c}}^{\prime}$ truly be the Hamiltonian of an OCP, the background and counterions clearly must occupy the same volume. In fact, were the background to occupy a different volume (e.g., the total volume $V$ ) then the effective Hamiltonian would contain terms that are formally infinite (see below), although identically cancelling, associated with the long-range Coulomb interaction. Thus, the background is taken to be excluded - along with the counterions - from the macroion cores, its density equaling the effective counterion density $n_{\mathrm{c}}$.

The background energy is then given explicitly by [22]

$$
E_{\mathrm{b}}=-\frac{1}{2} n_{\mathrm{c}}^{2} \int_{V_{\mathrm{f}}} \mathrm{d} \mathbf{r} \int_{V_{\mathrm{f}}} \mathrm{d} \mathbf{r}^{\prime} \frac{z^{2} e^{2}}{\epsilon\left|\mathbf{r}-\mathbf{r}^{\prime}\right|}=-\frac{1}{2} N_{\mathrm{c}} n_{\mathrm{c}} \hat{v}_{\mathrm{cc}}(0),
$$

where

$$
\hat{v}_{\mathrm{cc}}(0)=\int_{V_{\mathrm{f}}} \mathrm{d} \mathbf{r} \frac{z^{2} e^{2}}{\epsilon r}=\lim _{k \rightarrow 0}\left(\frac{4 \pi z^{2} e^{2}}{\epsilon k^{2}}\right)
$$

is the $k \rightarrow 0$ limit of the Fourier transform of $v_{\mathrm{cc}}(r)$. The infinity arising from $E_{\mathrm{b}}$ will be seen below to be formally cancelled by an identical infinity in $H_{\mathrm{mc}}$.

\subsection{Linear Response Approximation}

Thus far, the theory is exact, within the primitive model. The challenge lies ahead in calculating the counterion free energy [Eq. (8)]. One proposed approach 12 invokes density-functional theory to approximate $F_{\mathrm{c}}$, regarded as a functional of the counterion density, by expanding in a functional Taylor series about a uniform counterion OCP. An alternative strategy [16, 17], inspired by the pseudopotential theory of metals, is

to formally regard $H_{\mathrm{mc}}^{\prime}$ as an "external" potential acting upon a counterion OCP and approximate $F_{\mathrm{c}}$ by perturbation theory. Following the latter strategy[22], we write

$$
F_{\mathrm{c}}=F_{\mathrm{OCP}}+\int_{0}^{1} \mathrm{~d} \lambda\left\langle H_{\mathrm{mc}}^{\prime}\right\rangle_{\lambda}
$$


where

$$
F_{\mathrm{OCP}}=-k_{\mathrm{B}} T \ln \left\langle\exp \left(-H_{\mathrm{c}}^{\prime} / k_{\mathrm{B}} T\right)\right\rangle_{\mathrm{c}}
$$

is the free energy of the reference counterion OCP, occupying a volume $V_{\mathrm{f}}$, in the presence of neutral hard-sphere macroions. The integral over $\lambda$ in Eq. (12) physically corresponds to an adiabatic charging of the macroions from neutral to fully-charged spheres. The ensemble average \langle\rangle$_{\lambda}$ represents an average with respect to the distribution function of a system whose macroions are identically configured but carry a charge $\lambda Z$.

Further progress is facilitated by expressing $\left\langle H_{\mathrm{mc}}^{\prime}\right\rangle_{\lambda}$ in terms of Fourier components of the macroion and counterion densities and of the macroion-counterion interaction, according to

$$
\left\langle H_{\mathrm{mc}}^{\prime}\right\rangle_{\lambda}=\frac{1}{V_{\mathrm{f}}} \sum_{\mathbf{k} \neq 0} \hat{v}_{\mathrm{mc}}(k)\left\langle\hat{\rho}_{\mathrm{c}}(\mathbf{k})\right\rangle_{\lambda} \hat{\rho}_{\mathrm{m}}(-\mathbf{k})+\frac{1}{V_{\mathrm{f}}} \lim _{k \rightarrow 0}\left[\hat{v}_{\mathrm{mc}}(k)\left\langle\hat{\rho}_{\mathrm{c}}(\mathbf{k})\right\rangle_{\lambda} \hat{\rho}_{\mathrm{m}}(-\mathbf{k})\right]-E_{\mathrm{b}}
$$

Evidently $\left\langle H_{\mathrm{mc}}^{\prime}\right\rangle_{\lambda}$ depends through $\hat{\rho}_{\mathrm{c}}(\mathbf{k})$ upon the response of the counterions to the macroion charge density. Regarding the macroion charge as imposing an external potential on the counterions, the counterion density may be expressed in the form [23]

$$
\begin{aligned}
\hat{\rho}_{\mathrm{c}}(\mathbf{k}) & =\chi^{(1)}(k) \hat{v}_{\mathrm{mc}}(k) \hat{\rho}_{\mathrm{m}}(\mathbf{k})+\frac{1}{V_{\mathrm{f}}} \sum_{\mathbf{q}} \chi^{(2)}(\mathbf{q}, \mathbf{k}-\mathbf{q}) \hat{v}_{\mathrm{mc}}(q) \hat{v}_{\mathrm{mc}}(|\mathbf{k}-\mathbf{q}|) \hat{\rho}_{\mathrm{m}}(\mathbf{q}) \hat{\rho}_{\mathrm{m}}(\mathbf{k}-\mathbf{q}) \\
& +\cdots,
\end{aligned}
$$

where $\chi^{(i)}$ is the $i^{\text {th }}$ member of a hierarchy of response functions of the reference counterion OCP. Here, as in ref. [16], we adopt the simplest nontrivial approximation and assume that the counterions respond linearly to the macroion charges. Although its range of validity is uncertain, linearization is expected to be justified for sufficiently dilute suspensions and weakly charged macroions. Thus we take

$$
\left\langle\hat{\rho}_{\mathrm{c}}(\mathbf{k})\right\rangle_{\lambda}=\chi(k) \lambda \hat{v}_{\mathrm{mc}}(k) \hat{\rho}_{\mathrm{m}}(\mathbf{k}), \quad k \neq 0
$$

where $\chi(k) \equiv \chi^{(1)}(k)$ is the linear response function. Note that for $k=0$ there is no response, since $\hat{\rho}_{\mathrm{c}}(0)=N_{\mathrm{c}}$ is fixed by the number of counterions. Substituting Eqs. (16) and (14) into Eq. (12) and integrating over $\lambda$, the counterion free energy is given to second order in the macroion-counterion interaction by

$F_{\mathrm{c}}=F_{\mathrm{OCP}}+\frac{1}{2 V_{\mathrm{f}}} \sum_{\mathbf{k} \neq 0} \chi(k)\left[\hat{v}_{\mathrm{mc}}(k)\right]^{2} \hat{\rho}_{\mathrm{m}}(\mathbf{k}) \hat{\rho}_{\mathrm{m}}(-\mathbf{k})+n_{\mathrm{c}} \lim _{k \rightarrow 0}\left[N_{\mathrm{m}} \hat{v}_{\mathrm{mc}}(k)+\frac{N_{\mathrm{c}}}{2} \hat{v}_{\mathrm{cc}}(k)\right] .(17$

Correspondingly, the effective Hamiltonian takes the form

$$
\begin{aligned}
H_{\mathrm{eff}} & =K_{\mathrm{m}}+\frac{1}{2} \sum_{\substack{i, j=1 \\
i \neq j}}^{N_{\mathrm{m}}} v_{\mathrm{HS}}\left(\left|\mathbf{R}_{i}-\mathbf{R}_{j}\right|\right)+\frac{1}{2 V_{\mathrm{f}}} \sum_{\mathbf{k}} \hat{v}_{\mathrm{mm}}(k)\left[\hat{\rho}_{\mathrm{m}}(\mathbf{k}) \hat{\rho}_{\mathrm{m}}(-\mathbf{k})-N_{\mathrm{m}}\right] \\
& +F_{\mathrm{OCP}}+\frac{1}{2 V_{\mathrm{f}}} \sum_{\mathbf{k}} \chi(k)\left[\hat{v}_{\mathrm{mc}}(k)\right]^{2} \hat{\rho}_{\mathrm{m}}(\mathbf{k}) \hat{\rho}_{\mathrm{m}}(-\mathbf{k}) \\
& +n_{\mathrm{c}} \lim _{k \rightarrow 0}\left[-\frac{z N_{\mathrm{m}}}{2 Z} \chi(k)\left[\hat{v}_{\mathrm{mc}}(k)\right]^{2}+N_{\mathrm{m}} \hat{v}_{\mathrm{mc}}(k)+\frac{N_{\mathrm{c}}}{2} \hat{v}_{\mathrm{cc}}(k)\right] .
\end{aligned}
$$


Notice, however, that Eq. (18) may be restructured and written in the form

$$
\begin{aligned}
H_{\mathrm{eff}} & =K_{\mathrm{m}}+\frac{1}{2} \sum_{\substack{i, j=1 \\
i \neq j}}^{N_{\mathrm{m}}} v_{\mathrm{HS}}\left(\left|\mathbf{R}_{i}-\mathbf{R}_{j}\right|\right)+\frac{1}{2 V_{\mathrm{f}}} \sum_{\mathbf{k}} \hat{v}_{\mathrm{eff}}(k)\left[\hat{\rho}_{\mathrm{m}}(\mathbf{k}) \hat{\rho}_{\mathrm{m}}(-\mathbf{k})-N_{\mathrm{m}}\right]+E_{\mathrm{o}} \\
& =K_{\mathrm{m}}+\frac{1}{2} \sum_{\substack{i, j=1 \\
i \neq j}}^{N_{\mathrm{m}}}\left[v_{\mathrm{HS}}\left(\left|\mathbf{R}_{i}-\mathbf{R}_{j}\right|\right)+v_{\mathrm{eff}}\left(\left|\mathbf{R}_{i}-\mathbf{R}_{j}\right|\right)\right]+E_{\mathrm{o}},
\end{aligned}
$$

where

$$
\hat{v}_{\text {eff }}(k)=\hat{v}_{\text {mm }}(k)+\hat{v}_{\text {ind }}(k)
$$

may be interpreted as an effective electrostatic pair potential between pseudo-macroions, being the sum of the bare Coulomb potential and an induced potential

$$
\hat{v}_{\text {ind }}(k)=\chi(k)\left[\hat{v}_{\mathrm{mc}}(k)\right]^{2} \text {. }
$$

The final term in Eq. (19),

$$
E_{\mathrm{o}}=F_{\mathrm{OCP}}+\frac{N_{\mathrm{m}}}{2} \lim _{r \rightarrow 0} v_{\text {ind }}(r)+n_{\mathrm{c}} N_{\mathrm{m}} \lim _{k \rightarrow 0}\left[-\frac{z}{2 Z} \hat{v}_{\text {ind }}(k)+\hat{v}_{\mathrm{mc}}(k)+\frac{Z}{2 z} \hat{v}_{\mathrm{cc}}(k)\right],
$$

is the volume energy, which is a natural and inevitable consequence of the reduction to an equivalent one-component system. Although having no explicit dependence on the macroion positions (see below), $E_{\mathrm{o}}$ evidently depends on the average density of macroions and thus can make a significant contribution to the total free energy of the system. It must be emphasized that the above expressions for the effective pair potential and the volume energy are identical to expressions derived from the pseudopotential theory of metals [19, 22, 24] if one substitutes for $F_{\mathrm{OCP}}$ and $\chi(k)$, respectively, the energy and linear response function of the uniform electron gas, and for $\hat{v}_{\mathrm{mc}}(k)$ the electron-ion pseudopotential.

To summarize thus far, starting from the primitive model of charge-stabilized colloids, formally reducing the two-component macroion-counterion mixture to an equivalent one-component system of pseudo-macroions, and applying a linear response approximation to the counterion density, we have obtained expressions for both an effective electrostatic pair interaction [Eqs. (20) and (21)] and an associated volume energy [Eq. (22)]. Practical calculations still require specification of (1) the reference OCP free energy $F_{\mathrm{OCP}},(2)$ the OCP linear response function $\chi(k)$, and $(3)$ the macroioncounterion interaction $\hat{v}_{\text {mc }}(k)$. Below each of these is considered in turn.

It is important first to note that by associating the hard-sphere part of the total macroion-counterion interaction with the counterion Hamiltonian [Eq. (3)] - necessary, since response theory does not apply to hard-sphere interactions - the reference OCP is confined to the free volume between the macroion cores. As a consequence, the OCP is not strictly uniform since, in principle, the boundary conditions may induce nonuniformity. Determining the free energy of such a system in general poses a nontrivial task. In practice, however, counterion densities are usually low enough that the OCP may be assumed to be essentially uniform, except perhaps near contact with a macroion surface. 
Now, for typical macroion charges and concentrations, the OCP is so weakly coupled (unlike its electronic counterpart in metals) that its free energy is dominated by the ideal-gas entropic component. Therefore, ignoring correlations between counterions $\mathbb{1 2}$, 14, 20], an accurate approximation is

$$
F_{\mathrm{OCP}} \simeq k_{\mathrm{B}} T N_{\mathrm{c}}\left[\ln \left(n_{\mathrm{c}} \Lambda^{3}\right)-1\right]=k_{\mathrm{B}} T N_{\mathrm{c}}\left[\ln \left(\frac{(Z / z) n_{\mathrm{m}} \Lambda^{3}}{1-\eta}\right)-1\right],
$$

where $\Lambda$ is the thermal de Broglie wavelength and $n_{\mathrm{m}} \equiv N_{\mathrm{m}} / V$ is the average number density of macroions, the last equality following from the constraint of global charge neutrality.

The linear response function is directly related to the corresponding static structure factor $S(k)$ via

$$
\chi(k)=-\beta n_{\mathrm{c}} S(k)=-\frac{\beta n_{\mathrm{c}}}{1-n_{\mathrm{c}} \hat{c}(k)},
$$

where $\beta \equiv 1 / k_{\mathrm{B}} T$ and $\hat{c}(k)$ is the Fourier transform of the direct correlation function $c(r)$. Specifying $\chi(k)$ is therefore equivalent to specifying $\hat{c}(k)$. For a weakly coupled OCP, a convenient and reasonable approximation for $c(r)$ is given by the mean spherical approximation (MSA). This amounts to setting $c(r)$ equal to its asymptotic $(r \rightarrow \infty)$ limit $c(r) \simeq-\beta v_{\mathrm{cc}}(r)$ for all $r$. As a result,

$$
\hat{c}(k) \simeq-\beta \hat{v}_{\mathrm{cc}}(k)=-\frac{4 \pi \beta z^{2} e^{2}}{\epsilon k^{2}} .
$$

Substitution of Eq. (25) into Eq. (24) then yields [16]

$$
\chi(k)=-\frac{\beta n_{\mathrm{c}}}{1+\kappa^{2} / k^{2}},
$$

where

$$
\kappa \equiv\left(\frac{4 \pi n_{\mathrm{c}} z^{2} e^{2}}{\epsilon k_{\mathrm{B}} T}\right)^{1 / 2}=\left(\frac{4 \pi n_{\mathrm{c}}^{(o)} z^{2} e^{2}}{(1-\eta) \epsilon k_{\mathrm{B}} T}\right)^{1 / 2}
$$

As will be seen below, the parameter $\kappa$ plays the role of an inverse screening length in the counterion density profile and in the effective pair interaction.

Finally, specifying the macroion-counterion interaction amounts to determining the value of the parameter $\alpha$ in Eq. (5) that will ensure a vanishing counterion density inside the macroion cores. This in turn requires a calculation of the real-space counterion density profile, the details of which are described in the next section.

\section{Results}

\subsection{Counterion Density Profile}

The real-space counterion density profile $\rho_{\mathrm{c}}(\mathbf{r})$ may be determined from Eqs. (5), (16), and (26). First, Fourier transforming Eq. (5) yields

$$
\hat{v}_{\mathrm{mc}}(k)=-\frac{4 \pi Z z e^{2}}{\epsilon k^{2}}\left[(1-\alpha) \cos (k \sigma / 2)+\alpha \frac{\sin (k \sigma / 2)}{k \sigma / 2}\right] .
$$


Next, substituting Eqs. (26) and (28) into Eq. (16) gives the intermediate result

$\hat{\rho}_{\mathrm{c}}(\mathbf{k})=\frac{Z}{z}\left(\frac{\kappa^{2}}{k^{2}+\kappa^{2}}\right)\left[(1-\alpha) \cos (k \sigma / 2)+\alpha \frac{\sin (k \sigma / 2)}{k \sigma / 2}\right] \sum_{\mathbf{R}} \exp (i \mathbf{k} \cdot \mathbf{R})$,

where the sum is over the positions $\mathbf{R}$ of the macroions. For simplicity, we consider the density profile around a single macroion located at the origin $(\mathbf{R}=0)$, assuming all other macroions to be far away $(\kappa R \gg 1)$. This is equivalent to retaining only the $\mathbf{R}=0$ term in the summation, in which case $\hat{\rho}_{\mathrm{c}}(\mathbf{k})$ is a function only of $k$ and $\rho_{\mathrm{c}}(\mathbf{r})$ is a function only of the radial distance $r$. Now inverse Fourier transforming Eq. (29) yields $\rho_{\mathrm{c}}(r)= \begin{cases}\frac{Z}{z} \frac{\kappa^{2}}{4 \pi}\left[(1-\alpha) \cosh (\kappa \sigma / 2)+\alpha \frac{\sinh (\kappa \sigma / 2)}{\kappa \sigma / 2}\right] \frac{\exp (-\kappa r)}{r}, & r>\sigma / 2 \\ \frac{Z}{z} \frac{\kappa^{2}}{4 \pi}\left(-1+\alpha+\frac{\alpha}{\kappa \sigma / 2}\right) \mathrm{e}^{-\kappa \sigma / 2} \frac{\sinh (\kappa r)}{r}, & r<\sigma / 2 .\end{cases}$

Vanishing of $\rho_{\mathrm{c}}(r)$ for $r<\sigma / 2$ is evidently ensured by setting

$$
\alpha=\frac{\kappa \sigma / 2}{1+\kappa \sigma / 2} \text {. }
$$

Finally, substituting this expression for $\alpha$ back into Eq. (30) gives the result

$$
\rho_{\mathrm{c}}(r)=\frac{Z}{z} \frac{\kappa^{2}}{4 \pi}\left(\frac{\mathrm{e}^{\kappa \sigma / 2}}{1+\kappa \sigma / 2}\right) \frac{\mathrm{e}^{-\kappa r}}{r}, \quad r>\sigma / 2,
$$

which is automatically normalized to the correct number of counterions per macroion $(Z / z)$. This expression for the counterion density profile around a single macroion is recognized to be of precisely the same form as the Debye-Hückel expression for the density of electrolyte ions around a macroion [四], where $\kappa$ is the inverse Debye screening length. A notable distinction lies, however, in the definition of $\kappa$. Whereas our $\kappa$ [Eq. (27)] depends on the average effective counterion density $n_{\mathrm{c}}$ in the volume unoccupied by macroions, the Debye-Hückel $\kappa$ depends rather on the nominal bulk density of electrolyte ions. The importance of redefining the usual $\kappa$ in this way - a result that emerges naturally from the response approach - has been stressed also by Russel and coworkers [25].

In passing, two remarks are in order. First, determining the counterion density profile in the presence of two or more closely spaced macroions will evidently require a more general form for the macroion-counterion core interaction than the simple constant chosen in Eq. (5). Second, it may be instructive to compare the macroioncounterion interaction with its metallic counterpart, the electron-ion pseudopotential. A popular and successful form of the latter is the empty-hole pseudopotential [26], which is Coulombic at long range but precisely zero inside a certain core radius. In contrast, setting $v_{\text {mc }}(r)$ to zero for $r<\sigma / 2$ would result in a nonvanishing counterion density inside the macroion cores. A distinction between the metallic and colloidal cases lies in the fact that, while counterions are strictly excluded from macroion cores, electrons may at least partially penetrate metallic ion cores. 


\subsection{Effective Pair Interaction and Volume Energy}

We are now in a position to derive the main results of the paper. Considering first the effective electrostatic pair interaction between pseudo-macroions, we proceed by substituting Eq. (31) into Eq. (28), obtaining

$$
\hat{v}_{\mathrm{mc}}(k)=-\frac{4 \pi Z z e^{2}}{\epsilon k^{2}}\left(\frac{1}{1+\kappa \sigma / 2}\right)\left[\cos (k \sigma / 2)+\kappa \frac{\sin (k \sigma / 2)}{k}\right] .
$$

Next substituting Eqs. (26) and (33) into Eq. (21) yields

$\hat{v}_{\text {ind }}(k)=-\frac{2 \pi Z^{2} e^{2}}{\epsilon k^{2}}\left(\frac{1}{1+\kappa \sigma / 2}\right)^{2}\left(\frac{\kappa^{2}}{k^{2}+\kappa^{2}}\right)\left[1+\cos (k \sigma)+2 \kappa \frac{\sin (k \sigma)}{k}+\kappa^{2} \frac{1-\cos (k \sigma)}{k^{2}}\right]$.

Fourier transformation of Eq. (34) is a straightforward, if tedious, calculation, with the result

$$
v_{\text {ind }}(r)= \begin{cases}\frac{Z^{2} e^{2}}{\epsilon}\left(\frac{\mathrm{e}^{\kappa \sigma / 2}}{1+\kappa \sigma / 2}\right)^{2} \frac{\mathrm{e}^{-\kappa r}}{r}-\frac{Z^{2} e^{2}}{\epsilon r}, & r>\sigma \\ -\frac{Z^{2} e^{2}}{2 \epsilon r}\left(\frac{1}{1+\kappa \sigma / 2}\right)^{2}\left[(2+\kappa \sigma) \kappa r-\frac{1}{2} \kappa^{2} r^{2}\right], & r<\sigma .\end{cases}
$$

Finally, substituting Eq. (35) into the Fourier transform of Eq. (20), we obtain an explicit expression for the real-space form of the effective electrostatic pair potential:

$$
v_{\text {eff }}(r)=v_{\mathrm{mm}}(r)+v_{\text {ind }}(r)=\frac{Z^{2} e^{2}}{\epsilon}\left(\frac{\mathrm{e}^{\kappa \sigma / 2}}{1+\kappa \sigma / 2}\right)^{2} \frac{\mathrm{e}^{-\kappa r}}{r}, \quad r>\sigma .
$$

This result is identical in form to the electrostatic part of the familiar DLVO effective pair potential [5], which is usually derived from the Poisson-Boltzmann equation. The only difference between our potential and the DLVO potential lies in the definition of $\kappa$ [Eq. (27)], which here involves the effective counterion density $n_{\mathrm{c}}$.

The volume energy now may be explicitly determined from Eq. (22). It follows immediately from Eq. (35) that

$$
\lim _{r \rightarrow 0} v_{\text {ind }}(r)=-\frac{Z^{2} e^{2}}{\epsilon} \frac{\kappa}{1+\kappa \sigma / 2}
$$

from Eq. (34) that

$$
\lim _{k \rightarrow 0} \hat{v}_{\text {ind }}(k)=-\left(\frac{Z}{z}\right)^{2} \hat{v}_{\text {cc }}(0)+\frac{4 \pi Z^{2} e^{2}}{\epsilon \kappa^{2}}+\frac{\pi Z^{2} e^{2} \sigma^{2}}{\epsilon}\left(\frac{1}{1+\kappa \sigma / 2}\right)^{2}\left(1+\frac{2}{3} \kappa \sigma+\frac{1}{12} \kappa^{2} \sigma^{2}\right),
$$

and from Eq. (33) that

$$
\lim _{k \rightarrow 0} \hat{v}_{\mathrm{mc}}(k)=-\frac{Z}{z} \hat{v}_{\mathrm{cc}}(0)+\frac{\pi Z z e^{2}}{2 \epsilon}\left(\frac{1}{1+\kappa \sigma / 2}\right)\left(1+\frac{1}{6} \kappa \sigma\right) .
$$

Substituting Eqs. (37), (38), and (39) into Eq. (22), we obtain the following result for the volume energy:

$$
E_{\mathrm{o}}=F_{\mathrm{OCP}}-N_{\mathrm{m}} \frac{Z^{2} e^{2}}{2 \epsilon} \frac{\kappa}{1+\kappa \sigma / 2}-N_{\mathrm{m}} \frac{Z k_{\mathrm{B}} T}{2 z} .
$$

The first term on the right side of Eq. (40) is the OCP free energy, discussed in the previous section. The second term, which depends implicitly on the macroion 
density through the parameter $\kappa$, may be given a physical interpretation as one half the electrostatic energy associated with a single pseudo-macroion, composed of a macroion surrounded by its own screening cloud of counterions [12, 24]. The final term, corresponding to the $k \rightarrow 0$ limit in Eq. (22), contributes a density-independent constant to the free energy per macroion and hence has no influence on thermodynamic phase transitions at zero salt concentration. At finite salt concentration, however, the corresponding term cannot be ignored [13, 14, 21].

\section{Discussion and Conclusions}

It is important to point out some limitations of the theory and the results presented above. First of all, the assumption of linear response of the counterions is strictly valid only for dilute suspensions of weakly charged macroions. Whether the linear response approximation remains valid at higher concentrations - in particular, concentrations for which excluded volume effects begin to play a role - is an interesting and open question. In this regard, it may be worth noting that Poisson-Boltzmann cell model calculations [8] and ab initio simulations [11] do support the general form of the screenedCoulomb pair potential at appreciable concentrations, albeit with renormalized DLVO parameters. Secondly, by considering only electrostatic and hard steric interactions, and ignoring short-range interactions between counterions and macroion surfaces, the theory cannot address the possibility of condensation of counterions onto the macroions and the consequences for effective macroion charges. Finally, no account is taken of correlations between charge fluctuations, either on the macroion surfaces or in the density distributions of counterions surrounding neighbouring macroions. For spherical macroions, Monte Carlo simulations and cell model calculations [27] suggest that such correlations make only a small contribution to the total free energy, at least at low salt concentrations. On the other hand, for rod-like macroions, correlated charge fluctuations may play a more significant role 28].

In summary, by applying a linear response approximation to the counterions surrounding charged monodisperse hard-sphere macroions in a colloidal suspension, we have derived two main results, namely an effective electrostatic pair interaction $v_{\text {eff }}(r)$ [Eq. (36)] and an associated volume energy $E_{\mathrm{o}}$ [Eq. (40)]. The total free energy of the system is ultimately the sum of the volume energy - whose physical origins are the counterion entropy and the macroion-counterion interaction energy - and the free energy of the equivalent one-component system of pseudo-macroions interacting via their effective pair interaction. Our expression for $v_{\text {eff }}(r)$ confirms that a linear response approximation, combined with the MSA for the response function, yields the familiar DLVO form of pair potential for spherical macroions, prefactors included [29]. This is not surprising, given that the DLVO potential also may be derived by linearizing the Poisson-Boltzmann equation. At the same time, however, our derivation indicates how excluded volume corrections may be incorporated through the density dependence of the inverse screening length $\kappa$, by substituting for the nominal counterion density $n_{\mathrm{c}}^{(o)}$ the 
effective density $n_{\mathrm{c}}$ of counterions occupying the free volume between macroion cores.

Our expression for the volume energy, which quantitatively exhibits the dependence on the macroion density, confirms the necessity of including $E_{\mathrm{o}}$ in calculating thermodynamic properties from the free energy [16]. In particular, the phase behaviour of deionized suspensions of highly charged macroions have been shown to depend sensitively on the volume energy [12, 13, 14, 20]. Bulk pressure and elastic constants are expected to be similarly sensitive. Furthermore, our expression for $E_{\mathrm{o}}$ is consistent with that obtained by van Roij et al [13] from an alternative density-functional approach. The sole distinction is that our expression, which involves the effective counterion density $n_{\mathrm{c}}$, incorporates excluded volume corrections, at least in an approximate fashion. Although not likely of significant consequence at the small volume fractions considered in Refs. [12] and [13], such effects may become important at higher concentrations [20].

In conclusion, the linear response approach of Silbert et al [16, 17] offers a powerful tool for investigating effective electrostatic interactions in charge-stabilized colloidal suspensions. As demonstrated here, a consistent extension to finite-sized macroions leads directly to (1) an effective pair interaction between pseudo-macroions having precisely the DLVO screened-Coulomb form, but with a modified inverse screening length that incorporates excluded volume corrections, and (2) a density-dependent volume energy that can make a significant contribution to the total free energy of salt-free suspensions. By including the next higher-order response function $\left[\chi^{(2)}\right.$ in Eq. (15)], the approach can be straightforwardly generalized to include nonlinear response of microions and thereby used to assess the importance of effective threebody interactions [11]. This is equivalent to approximating the counterion free energy [Eq. (12)] in perturbation theory to third order in the macroion-counterion interaction. An expression for an effective triplet interaction already has been derived from a density-functional approach [30. It remains, however, to analyse the corrections that are entailed both to the volume energy and to the effective pair interaction, and to explore the implications for thermodynamic properties. Experience from ab initio simulations [11] and from the realm of metals [31] suggests that many-body effects become significant at sufficiently high densities. Further outstanding issues are whether in bulk the effective pair interaction always retains its screened-Coulomb form, and whether near a boundary the interaction can ever become attractive [15. It is hoped that in future the response approach may help to resolve these important issues.

\section{Acknowledgments}

It is a pleasure to thank Anne M. Denton, Hartmut Löwen, Hartmut Graf, and Christos N. Likos for helpful discussions. 


\section{References}

[1] Hunter R J 1986 Foundations of Colloid Science (Oxford: Oxford University Press)

[2] Pusey P N 1991 in Liquids, Freezing and Glass Transition session 51 ed Hansen J-P, Levesque D, and Zinn-Justin J (Amsterdam: North-Holland)

[3] Pan G, Kesavamoorthy R, and Asher S A 1997 Phys. Rev. Lett. 783860

[4] Wijnhoven J E G J and Vos W L 1998 Science 281 802; Soukoulis C M ed 1996 Photonic Band Gap Materials (Dordrecht: Kluwer Academic)

[5] Derjaguin B V and Landau L 1941 Acta Physicochimica (USSR) 14 633; Verwey E J W and Overbeek J T G 1948 Theory of the Stability of Lyophobic Colloids (Amsterdam: Elsevier)

[6] Larsen A E and Grier D G 1997 Nature 385 230; Ito K, Yoshida H, and Ise N 1994 Science 263 66; Tata B V R, Rajalakshmi M, and Arora A K 1992 Phys. Rev. Lett. 693778

[7] Rowlinson J S 1984 Mol. Phys. 52567

[8] Alexander S, Chaikin P M, Grant P, Morales G J, and Pincus P 1984 J. Chem. Phys. 805776

[9] Stevens M J, Falk M L and Robbins M O 1996 J. Chem. Phys. 1045209

[10] Allahyarov E, D'Amico I and Löwen H 1998 Phys. Rev. Lett. 811334

[11] Löwen H, Madden P A and Hansen J-P 1992 Phys. Rev. Lett. 68 1081; 1993 J. Chem. Phys. 98 3275; Löwen H and Kramposthuber G 1993 Europhys. Lett. 23 673; Tehver R, Ancilotto F, Toigo F, Koplik J, and Banavar J R 1999 Phys. Rev. E 59 R1335

[12] van Roij R and Hansen J-P 1997 Phys. Rev. Lett. 793082

[13] van Roij R, Dijkstra M, and Hansen J-P 1999 Phys. Rev. E 592010

[14] Graf H and Löwen H 1998 Phys. Rev. E 575744

[15] Goulding D and Hansen J-P 1998 Mol. Phys. 95649

[16] Grimson M J and Silbert M 1991 Mol. Phys. 74397

[17] Canessa E, Grimson M J and Silbert M 1988 Mol. Phys. 64 1195; 1990 in Strongly Coupled Plasma Physics ed S Ichimaru (Elsevier/Yamada Science Foundation), p 675

[18] Harrison W A 1970 Pseudopotentials in the Theory of Metals (New York: Benjamin); Heine V 1970 Solid State Phys. 241

[19] Hafner J 1987 From Hamiltonians to Phase Diagrams (Berlin: Springer)

[20] Denton A R and Löwen H 1998 Phys. Rev. Lett. 81469

[21] Denton A R (unpublished)

[22] Hansen J-P and McDonald I R 1986 Theory of Simple Liquids $2^{\text {nd }}$ edition (London: Academic), Chap. 11

[23] Ashcroft N W and Stroud D 1978 Solid State Phys. 331

[24] Finnis M W 1974 J. Phys. F: Metal Phys. 41645

[25] Russel W B 1981 in Theory of Dispersed Multiphase Flow ed R E Meyer (New York: Academic); Russel W B and Benzing D W 1981 J. Coll. Int. Sci. 83 163; Gast A P, Russel W B and Hall C K 1986 J. Coll. Int. Sci. 109161

[26] Ashcroft N W 1966 Phys. Lett. 2348

[27] Woodward C E, Jönsson B, and Åkesson T 1988 J. Chem. Phys. 89 5145; Attard P, Kjellander R, and Mitchell D J 1988 J. Chem. Phys. 891664

[28] Ha B-Y and Liu A J 1997 Phys. Rev. Lett. 79 1289; 1998 Phys. Rev. E 586281

[29] The present results correct the prefactor in the effective pair potential for finite-sized macroions given in Ref. [16].

[30] Löwen H and Allahyarov E 1998 J. Phys.: Condens. Matter 104147

[31] Louis A A and Ashcroft N W 1998 Phys. Rev. Lett. 814456 